\title{
MRI of sciatic nerve tumours: correlation with histological findings
}

\author{
Molinier B ${ }^{1}$, Brauge $D^{2}$, Lapègue $\mathrm{F}^{1}$, Gandois $\mathrm{HC}^{1}$, Gomez-Brouchet $\mathrm{A}^{3}$, Sans $\mathrm{N}^{1}$ and Bilfeld $\mathrm{MF}^{*}$ \\ ${ }^{1}$ Department of Radiology, CHU Toulouse-Purpan, Place du docteur Baylac, Toulouse, France \\ ${ }^{2}$ Department of Neurosurgery, CHU Toulouse-Purpan, Place du docteur Baylac, Toulouse, France \\ ${ }^{3}$ Department of Anatomy and Pathology, Institut Universitaire du Cancer de Toulouse - Oncopôle, avenue Irène Joliot-Curie, Toulouse, France
}

\begin{abstract}
Objective: Describe the MRI signs suggestive of an aetiology for newly discovered sciatic nerve tumours.

Method: We retrospectively analysed 20 patients with 24 sciatic nerve tumours. Two observers described the preoperative MRI features of the lesions (size, shape, margins, signal, gadolinium uptake, location on the nerve, dissection of nerve fascicles) and the presence of oedema in the adjacent soft tissues.

Results: The 24 tumours analysed fell within 7 histological types: schwannomas, neurofibromas, plexiform neurofibromas, malignant peripheral nerve sheath tumour (MPNST), lymphomas, metastasis and perineurioma. Regarding the benign tumours $80 \%$ of the neurofibromas and only $25 \%$ of the schwannomas were centred relative to the nerve. All histological types (except perineurioma) had a heterogeneous signal and uptake. None of the benign lesions (0/17) and all the malignant lesions (7/7) had ill-defined margins and oedema surrounding the lesion. Nerve fascicle dissection was not present in the MPNST; this sign was found in $100 \%$ of the malignant tumours.
\end{abstract}

Conclusion: The size, shape, signal and contrast product uptake of sciatic nerve tumours cannot be used as diagnostic criteria. The presence of soft tissue oedema surrounding the lesion is suggestive of a malignant lesion. Nerve fascicle dissection is suggestive of non-neurogenic malignant tumours.

Abbreviation: MPNST: Malignant Peripheral Nerve Sheath Tumours; NF1: Neurofibromatosis Type I; MRI- Magnetic Resonance Imaging.

\section{Introduction}

Soft tissue tumours that develop from nerve structures form a heterogeneous set of benign and malignant lesions [1]. Most of these are nerve sheath tumours. Schwann cells, the main component of the endoneurum, perineurum and epineurum surrounding the axons, are most often the source of the tumour. The sciatic nerve is a one of the most common locations for lower limb even if it is a rare location [2]. The nerve entering and exiting the tumour is the signs most often suggestive of a neurogenic origin . Magnetic resonance imaging (MRI) is the imaging modality of choise to explore the pathology [3]. While ultrasonography provides information about the tumour's location, size and relationship with neighbouring structures, the deep placement of this nerve, particularly on its course from the anterior sciatic foramen to the piriformis muscle, makes it difficult to explore. Computed tomography provides similar information but does not allow for sufficient tissue characterisation [4]. MRI provides an accurate morphological analysis because of its excellent tissue contrast and high spatial resolution. Tissues can be characterised by analysing the lesion's signal [5]. MRI is the most sensitive and specific tool for differentiating between benign and malignant tumours of the peripheral nerve sheaths [6]. MRI is a guide for potential surgical procedures, based on a description of the involved anatomical compartments, and for postoperative follow-up.

The objective of this study was to describe the MRI signs suggestive of an aetiology for newly discovered sciatic nerve tumours and discuss them according literature data.

\section{Material and method}

This was a retrospective, single-centre study of cases recorded in a database used by the multidisciplinary study group for bone and soft tissue tumours at our healthcare facility, which is a national referral centre, between March 2001 and March 2016. This study was approved by our research ethics committee.

\section{Study population}

Twenty patients (11 men, 9 women), four of whom had bilateral lesions, ranging in age from 2 to 73 years (mean age: 41 years, SD 21) were included. The study population consisted of 24 tumours in 20 patients who had a lesion in the sciatic nerve on MRI and a histological diagnosis of biopsied tissue after the MRI had been done. The exception was cases of typical plexiform neurofibromas in the context of NF1 when histological proof was available from a lower-risk lesion (subcutaneous neurofibroma).

\section{MRI protocol}

The MRI protocol for evaluating the sciatic nerve at our facility consisted of axial T1-weighted spin-echo sequences $(\mathrm{TR} / \mathrm{TE}=575 / 10)$, coronal proton density fat-saturated (PD Fat Sat) sequences (TR/TE

${ }^{\star}$ Correspondence to: Bilfeld MF, Department of Radiology, CHU ToulousePurpan, place du Docteur Baylac, Toulouse, France, Tel: 330561772297; E-mail: faruch.m@chu-toulouse.fr

Key words: sciatic nerve, mri, tumour characterisation

Received: July 01, 2019; Accepted: July 08, 2019; Published: July 10, 2019 
$=4400 / 120)$ and axial T1-weighted fat-suppressed post-gadolinium sequences $(\mathrm{TR} / \mathrm{TE}=460 / 10)$. Other sequences and slice planes were carried out at the discretion of the radiologist in charge of the session. Because our facility recently changed its MRI unit, 14 patients were imaged using a $1.5 \mathrm{~T}$ unit (Optima MR360; GE Healthcare, WI, USA) and 8 with a $3 \mathrm{~T}$ unit (MAGNETOM ${ }^{\circ}$ Skyra; Siemens, Erlangen, Germany). A $16-\mathrm{cm}$ field of view was used with a 512 x 512 matrix. The slice thicknesses were $3 \mathrm{~mm}$ for coronal and sagittal planes and $5 \mathrm{~mm}$ for axial planes. The sequences in axial planes were centred over the lesion after completing the scout scan.

\section{Image analysis}

Two raters, a senior radiologist (MFB) with 10 years' experience in musculoskeletal imaging and a junior radiologist (BM) with 5 years' experience independently reviewed the sequences and described the features of the tumours. They were blinded to the patients' history and clinical signs. The MRI records were rendered anonymous and interpreted on a McKesson data archiving and retrieval workstation without any knowledge of the results of other imaging modalities or pathology data.

The following items were analysed on MRI:

- size of lesion (largest diameter in millimetres)

- number (single or multiple)

- shape (ovoid or irregular) and margins (well-defined or ill-defined) (Figures 1,2 and 3)
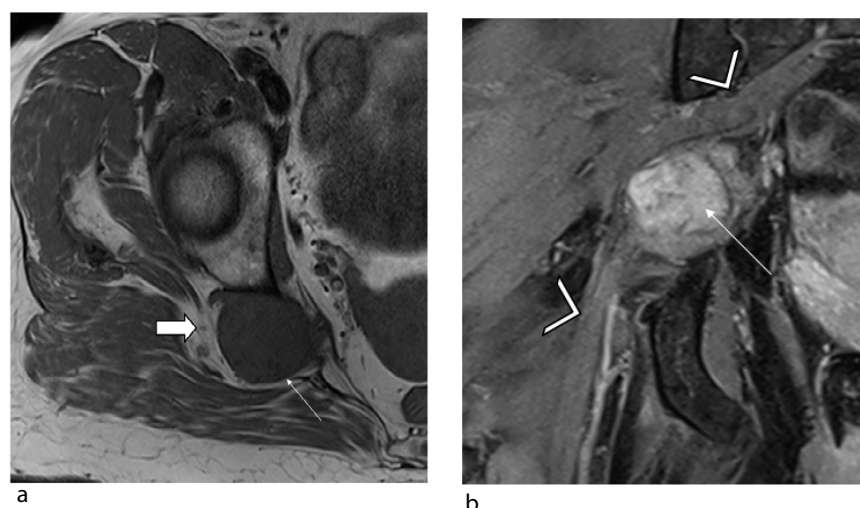

b

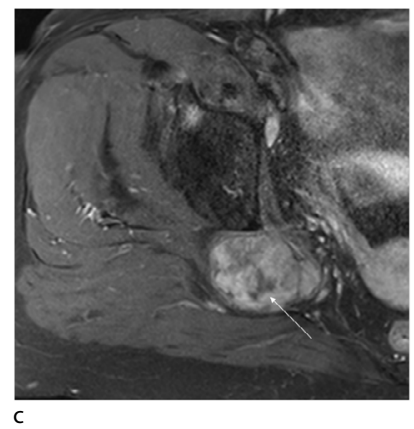

Figure 1. Female patient, 43 years old: schwannoma of the sciatic nerve at the right sciatic notch. (a) Axial T1 slice (TR/TE 850/11) showing a neurogenic lesion (arrow) with isosignal, well-defined margins and no spread to adjacent soft tissues (solid arrow) (b) Fluid-sensitive coronal slice (TR/TE 2200/34) showing the hyperintense lesion (arrow) and absence of target sign. The tumour is off-centred relative to the sciatic nerve (arrowhead). (c) Axial T1-weighted fat-suppressed post-gadolinium slice showing heterogeneous uptake in the tumour (arrow)
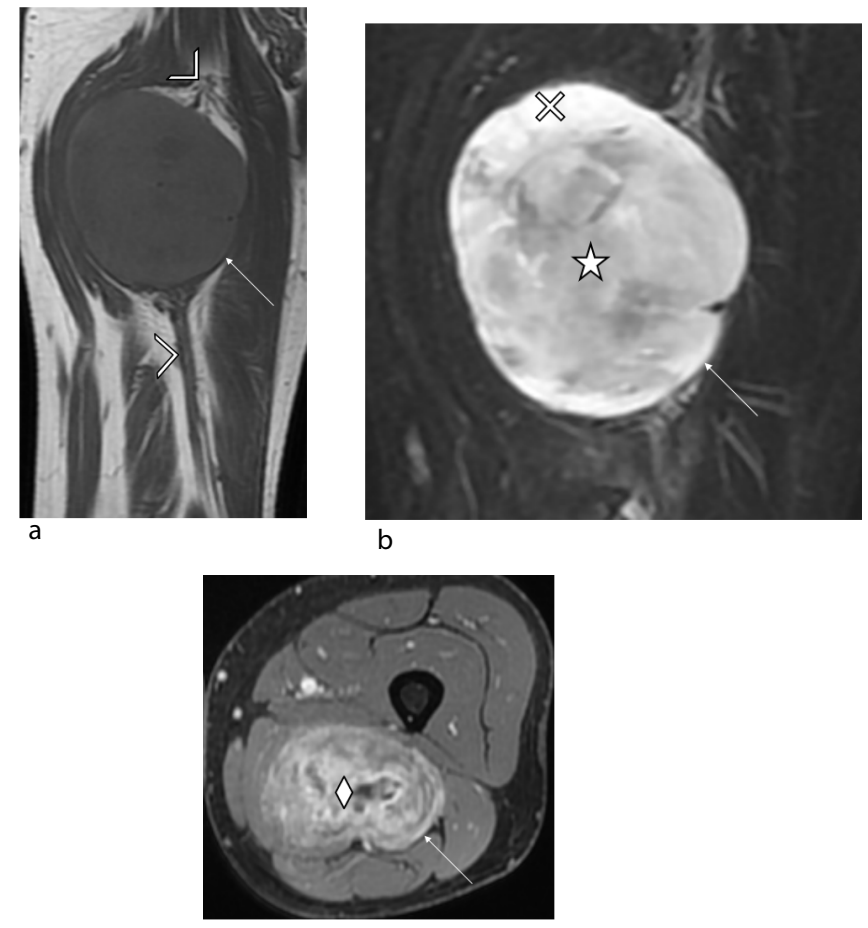

Figure 2. Male patient, 42 years old with no relevant medial history: neurofibroma of right sciatic nerve. (a) Coronal $\mathrm{T} 1$ slice (TR/TE 568/11) showing a neurogenic lesion (arrow) with isosignal, well-defined margins and no spread to adjacent soft tissues (solid arrow). The tumour is centred relative to the sciatic nerve (arrowhead). (b) Fluid-sensitive coronal slice (TR/TE 3998/72) showing the heterogeneous lesion (arrow) and target sign: central hypointensity (star) and peripheral hyperintensity (cross). (c) Axial T1-weighted fat-suppressed post-gadolinium slice showing heterogeneous uptake in the tumour (arrow) with predominantly central uptake (diamond)

- appearance of nerve above the lesion with potential nerve fascicle dissection (Figures 4 and 5)

- location relative to the nerve - centred or off-centred (Figure 6)

- signal intensity relative to adjacent muscle tissue on T1 and PD Fat Sat sequences and heterogeneity with possibility of the target sign (central hypointense and peripheral hyperintense signal)

- uptake after gadolinium injection

- invasion of soft tissues (considered present when greater uptake was seen in the tissues adjacent to the lesion on gadolinium-enhanced T1-weighted sequences).

\section{Statistical analysis}

The measurements made by the most experienced radiologist (MFB) were used in the descriptive analysis. The second radiologist's measurement $(\mathrm{BM})$ were used to assess measurement reproducibility. The descriptive analysis consisted of calculating the mean and standard deviation values. To evaluate the reproducibility of the findings, the interobserver agreement was determined by calculating Cohen's Kappa coefficient, which was interpreted as suggested by Landis et Koch [7] for categorial data and a student $t$ test was used for quantitative data. The statistical tests were carried out with Medcal software.

\section{Result}

All 20 patients had minor symptoms, such as sensory or motor disorders in the territory of the sciatic nerve. On clinical examination, 11 patients had a lump on the posterior aspect of the thigh. 


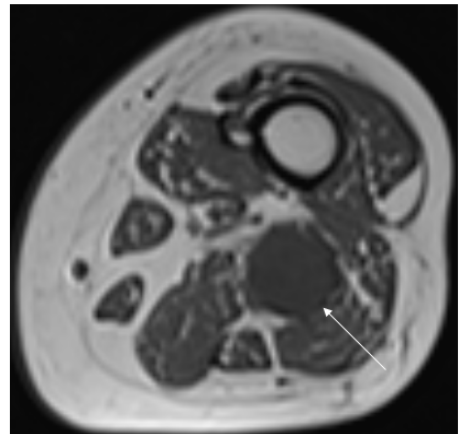

a

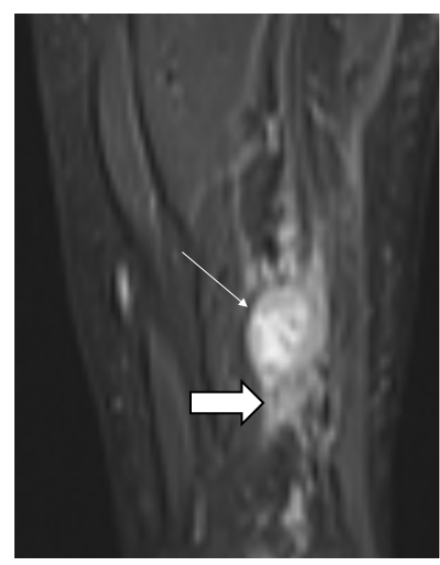

b

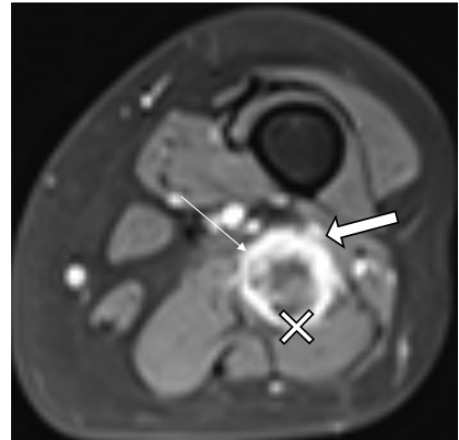

C

Fire 3. Female patient, 34 years old, suffering from neurofibromatosis type I who recently developed pain due to neurofibroma of the left sciatic nerve: malignant transformation to MPNST confirmed histologically. (a) Axial T1 slice (TR/TE 614/9) showing a neurogenic lesion (arrow) with T1 isosignal and ill-defined margins. (b) Coronal STIR slice (TR/TE 6531/32) showing lesion (arrow) with heterogeneous signal without target sign and spread to adjacent soft tissues (solid arrow). (c) Axial T1-weighted fat-suppressed post-gadolinium slice showing heterogeneous uptake in the tumour (arrow) with predominantly peripheral uptake (cross). The tumour has irregular borders (solid arrow).

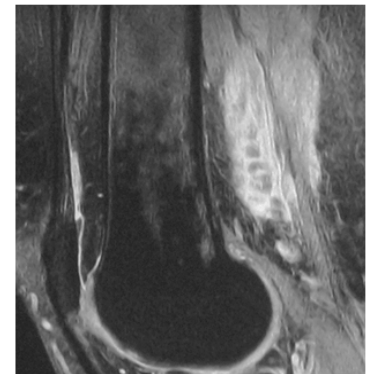

a

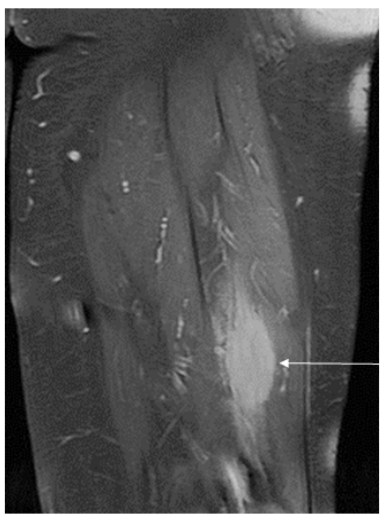

d

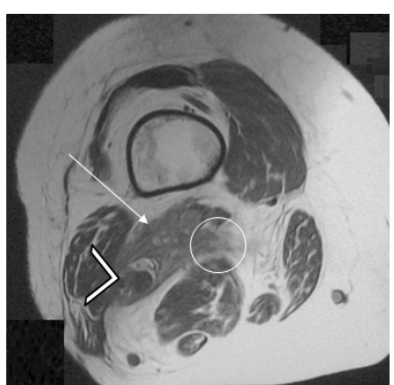

b

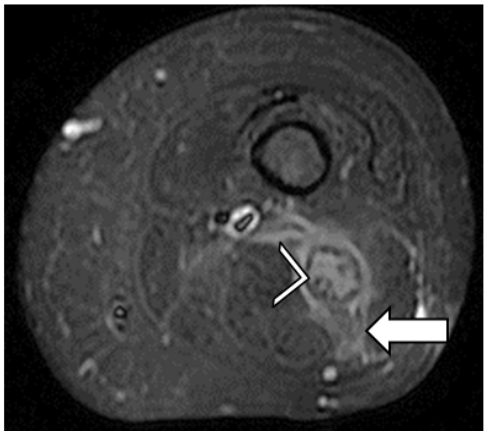

e

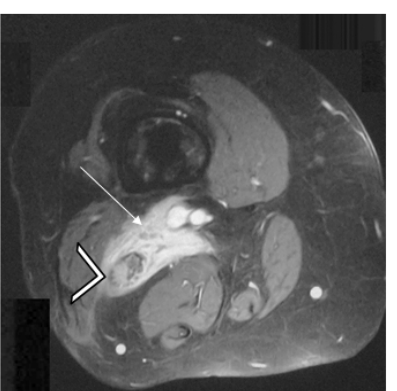

C

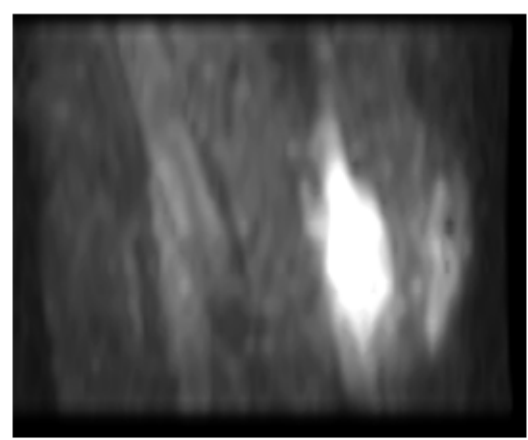

f

Figure 4. Female patient, 49 years old, lymphoma discovered (confirmed histologically) in the posterior area of the left thigh that invaded the sciatic nerve (a, b, c) during an assessment for knee pain. Intraneural metastasis of the contralateral sciatic nerve developed 1 year later (confirmed histologically) (d, e, f): (a) Sagittal proton density fat-saturated slice (TR/TE $2700 / 24$ ) showing the tumour (arrow) with heterogeneous signal and tumour invasion (hyperintensity) of the sciatic nerve (arrow head) leading to dissection of the nerve fascicles (hypointense signal). (b) Axial T1 slice (TR/TE 440/11) showing an irregular-shaped tumour (arrow) with ill-defined margins (circle) in the posterior region of the left thigh over the path of the sciatic nerve (arrowhead). (c) Axial T1-weighted fat-suppressed post-gadolinium slice showing homogeneous uptake in the tumour (arrow) with dissection of the sciatic nerve fascicles (arrowhead). (d, e) Coronal and axial slices from proton density fat-saturated sequences (TR/TE 2700/24) showing a fusiform tumour (arrow) of the sciatic nerve with invasion of the adjacent soft tissues (solid arrow), evidence of hematogenous spread to the contralateral side. Note the dissection of the sciatic nerve fascicles (hypointense signal) by the hyperintense infiltrating tumour (arrowhead) (f) Sagittal slice from diffusion-weighted b800 sequence showing hyperintensity, which confirms the lymphatic origin of the tumour 


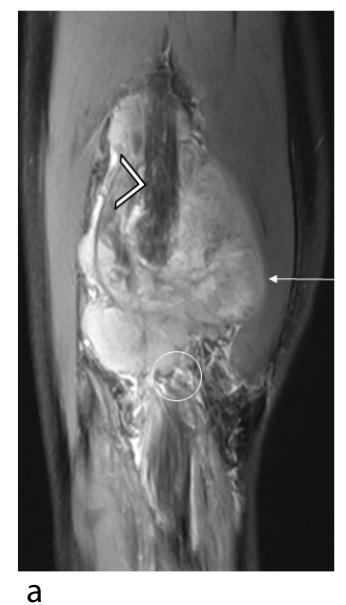

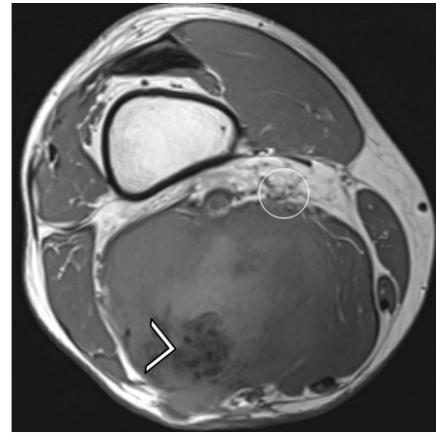

b

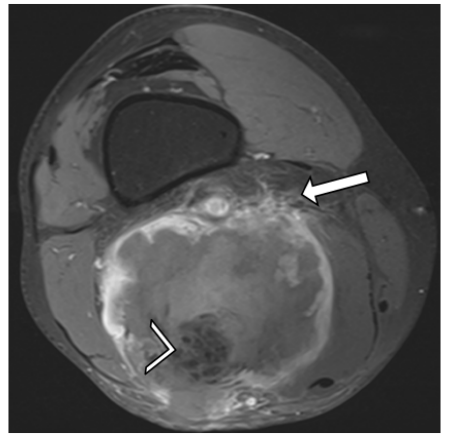

C

Figure 5. Male patient, 59 years old, suffering from pleural mesothelioma secondary to asbestos exposure. Recent appearance of lump in left thigh: intraneural metastasis of the pleural mesothelioma confirmed histologically. (a) Coronal proton density fat-saturated slice (TR/TE 3000/24) showing the tumour (arrow) with a heterogeneous signal and ill-defined margins (circle) but no target sign. Note the dissection of the hypointense sciatic nerve fascicles (arrowhead) by the hyperintense infiltrating tumour. (b) Axial T1 slice (TR/TE 900/12) showing a large tumour (arrow) centred on the left sciatic nerve (arrowhead) with a heterogeneous signal and ill-defined margins (circle). (c) Axial T1-weighted fat-suppressed post-gadolinium slice showing heterogeneous uptake in the tumour (arrow) with predominantly peripheral uptake and spread into soft tissues (solid arrow). Also note the dissection of the sciatic nerve fascicles (arrowhead).

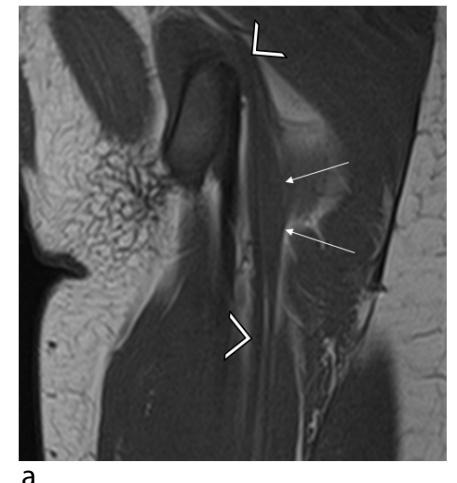

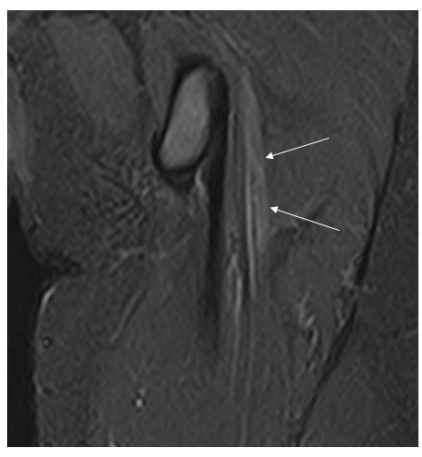

b

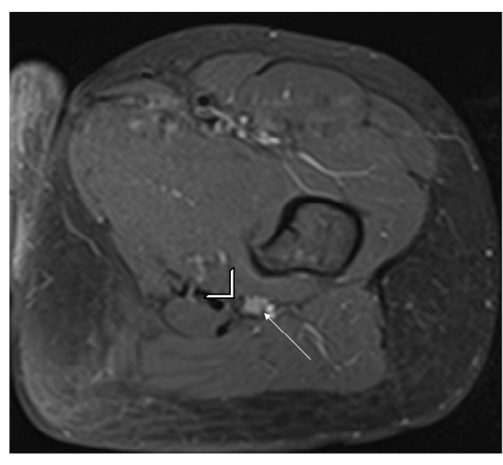

C

Figure 6. Young female patient (11 years old) with isolated deficit in the territory of the common peroneal nerve: intraneural perineurioma of the left sciatic nerve confirmed histologically (a, b) Coronal T1 (TR/TE 592/10) and coronal STIR (TR/TE 3445/15) slices showing local, crude hypertrophy of the sciatic nerve with T1 isosignal and STIR hyperintensity (arrow) with no spread to adjacent soft tissues. (c) Axial T1-weighted fat-suppressed post-gadolinium slice showing homogeneous uptake in the tumour (arrow) which is off-centred relative to the sciatic nerve (arrowhead).

For 17 of the 24 tumours, histological confirmation was available. The seven without direct pathology evidence were plexiform neurofibromas with pathognomonic appearance on imaging; these 7 cases had excised subcutaneous neurofibromas that were used to confirm their pathology.

Table 1 summarises the features of the patients, the location of the sciatic nerve lesions and whether there was one or multiple tumours. Table 2 summarises the MRI findings for each histological type of lesion.

The interobserver reproducibility was excellent: the Kappa coefficient ranged between 0.93 and 1.00 and Student $\mathrm{T}$ test were not significative for quantitative data with $\mathrm{p}>0,05$.

\section{Discussion}

This study was a retrospective MRI analysis of seven histological sciatic nerve tumour types. Knowing their MRI characteristics makes it possible to guide diagnosis upon discovery of a sciatic nerve lesion. Only plexiform neurofibromas can be conclusively diagnosed with imaging due to a specific sign: multinodal thickening of the nerve as it was described in previous study [8].

The size, shape, signal and uptake of a sciatic nerve tumour cannot be used as diagnostic criteria to predict the histological type.

In this study, the MPNST had a smaller size than 9 of the 10 isolated benign lesions. The lack of discriminatory power of size is consistent with several published reports showing that size cannot be used to differentiate between benign and malignant tumours in the case of sheath tumours, nor between neurogenic and non-neurogenic tumours [9]. This runs counter to many studies that use a size greater than $5 \mathrm{~cm}$ as a criterion for malignancy [10].

Shape did not predict whether a tumour was benign or malignant in our study; lymphomas have a regular shape and benign tumours of the nerve sheath can be irregular. Ogose et al. showed that $50 \%$ of malignant tumours have a regular shape [9].

In our study, almost all lesions had a heterogeneous signal. This conflicts with the idea that these criterion is associated with malignancy 
Table 1. Demographic characteristics of patients with number, size and pathological diagnosis of their sciatic nerve lesion

\begin{tabular}{|c|c|c|c|c|c|c|c|}
\hline Patient & Age & Sex & Context & Number & Size (mm) & Histological confirmation & Diagnosis \\
\hline 1 & 65 & M & - & 1 & NM & Surgical biopsy & Lymphoma \\
\hline 2 & 28 & M & NF1 & Bilateral & NM & Excision of subcutaneous lesion & Plexiform neurofibroma \\
\hline 3 & 49 & $\mathrm{~F}$ & - & $\begin{array}{c}\text { Bilateral } \\
\text { (metastasis left } \\
\text { side) }\end{array}$ & $\begin{array}{c}67 \text { (right) } \\
48 \text { (left) }\end{array}$ & Ultrasound-guided biopsy & Lymphoma \\
\hline 4 & 43 & M & - & 1 & 34 & Surgical excision & Schwannoma \\
\hline 5 & 27 & $\mathrm{~F}$ & - & 1 & 57 & Surgical excision & Schwannoma \\
\hline 6 & 2 & M & NF1 & 1 & NM & Excision of subcutaneous lesion & Plexiform neurofibroma \\
\hline 7 & 65 & $\mathrm{~F}$ & - & 1 & 55 & Surgical excision & Schwannoma \\
\hline 8 & 34 & $\mathrm{~F}$ & NF1 & 1 & 33 & Surgical excision & MPNST \\
\hline 9 & 35 & M & NF1 & Bilateral & $\begin{array}{l}163 \text { (right) } \\
109 \text { (left) }\end{array}$ & Ultrasound-guided biopsy & Neurofibroma \\
\hline 10 & 69 & $\mathrm{~F}$ & - & 1 & NM & Surgical biopsy & $\begin{array}{l}\text { Metastasis of urothelial } \\
\text { carcinoma }\end{array}$ \\
\hline 11 & 15 & M & NF1 & Bilateral & NM & Excision of subcutaneous lesion & Plexiform neurofibroma \\
\hline 12 & 15 & $\mathrm{~F}$ & NF1 & 1 & 13 & Excision of subcutaneous lesion & Neurofibroma \\
\hline 13 & 24 & M & NF1 & 1 & NM & Excision of subcutaneous lesion & Plexiform neurofibroma \\
\hline 14 & 59 & M & - & 1 & 113 & Ultrasound-guided biopsy & Metastasis of mesothelioma \\
\hline 15 & 16 & M & NF1 & 1 & NM & Excision of subcutaneous lesion & Plexiform neurofibroma \\
\hline 16 & 54 & M & - & 1 & 150 & Ultrasound-guided biopsy & Neurofibroma \\
\hline 17 & 42 & M & - & 1 & 100 & Ultrasound-guided biopsy & Neurofibroma \\
\hline 18 & 65 & $\mathrm{~F}$ & - & 1 & 53 & Surgical excision & Schwannoma \\
\hline 19 & 73 & $\mathrm{~F}$ & - & 1 & NM & Surgical biopsy & Lymphoma \\
\hline 20 & 12 & $\mathrm{~F}$ & - & 1 & 74 & Surgical biopsy & Perineurioma \\
\hline
\end{tabular}

NM: not measured

NF1: neurofibromatosis type I

MPNST: malignant peripheral nerve sheath tumours

Table 2. Clinical and MRI features of lesions by histological type

\begin{tabular}{|c|c|c|c|c|c|c|c|}
\hline Histological type & Schwannoma & Neurofibroma & $\begin{array}{c}\text { Plexiform } \\
\text { neurofibroma }\end{array}$ & MPNST & Lymphoma & Metastasis & Perineurioma \\
\hline Mean age & 50 years $(27-65)$ & 36 years $(15-54)$ & 17 years $(2-28)$ & 34 years & 62 years $(49-73)$ & 64 years $(59-69)$ & 12 years \\
\hline Context & $0 \%$ NF1 (0/4) & $50 \%$ NF1 $(2 / 4)$ & $100 \%$ NF1 (5/5) & $100 \%$ NF1 (1/1) & $0 \%$ NF1 $(0 / 3)$ & $0 \%$ NF1 $(0 / 2)$ & $0 \%$ NF1 $(0 / 1)$ \\
\hline $\begin{array}{l}\text { Pathological } \\
\text { appearance of nerve } \\
\text { above and below }\end{array}$ & $0 \%(0 / 4)$ & $0 \%(0 / 5)$ & $100 \%(7 / 7)$ & $100 \%(1 / 1)$ & $100 \%(4 / 4)$ & $100 \%(2 / 2)$ & $0 \%(0 / 1)$ \\
\hline $\begin{array}{c}\text { Location relative to } \\
\text { nerve }\end{array}$ & $75 \%$ off-centred (3/4) & $80 \%$ centred $(4 / 5)$ & $100 \%$ centred $(7 / 7)$ & $\begin{array}{c}100 \% \text { off-centred } \\
(1 / 1)\end{array}$ & $100 \%$ centred $(4 / 4)$ & $100 \%$ centred $(2 / 2)$ & $\begin{array}{c}100 \% \text { off-centred } \\
(1 / 1)\end{array}$ \\
\hline Average size & $\begin{array}{c}50 \mathrm{~mm}(34-57 ; \mathrm{SD}: \\
10.6)\end{array}$ & $\begin{array}{l}106 \text { mm (13-163; } \\
\text { SD: } 59)\end{array}$ & NM & $33 \mathrm{~mm}$ & $\begin{array}{c}58 \mathrm{~mm}(48-67 ; \mathrm{SD}: \\
13.4 ; 2 \mathrm{NM}\end{array}$ & $113 \mathrm{~mm}(1 \mathrm{NM})$ & $74 \mathrm{~mm}$ \\
\hline Irregular shape & $25 \%(1 / 4)$ & $20 \%(2 / 5)$ & $0 \%(0 / 7)$ & $0 \%(0 / 1)$ & $75 \%(3 / 4)$ & $100 \%(2 / 2)$ & $0 \%(0 / 1)$ \\
\hline Ill-defined margins & $0 \%(0 / 4)$ & $0 \%(0 / 5)$ & $0 \%(0 / 7)$ & $100 \%(1 / 1)$ & $100 \%(4 / 4)$ & $100 \%(2 / 2)$ & $0 \%(0 / 1)$ \\
\hline Heterogeneous signal & $75 \%(3 / 4)$ & $100 \%(5 / 5)$ & $86 \%(6 / 7)$ & $100 \%(1 / 1)$ & $75 \%(3 / 4)$ & $100 \%(2 / 2)$ & $0 \%(0 / 1)$ \\
\hline T1 & \multicolumn{7}{|c|}{$100 \%$ isosignal } \\
\hline T2 & \multicolumn{7}{|c|}{$100 \%$ hypersignal } \\
\hline Target sign & $25 \%(1 / 4)$ & $80 \%(4 / 5)$ & $86 \%(6 / 7)$ & $0 \%(0 / 1)$ & $0 \%(0 / 4)$ & $0 \%(0 / 2)$ & $0 \%(0 / 1)$ \\
\hline Gadolinium uptake & $\begin{array}{c}100 \%(4 / 4): \\
-0 \% \text { homogeneous } \\
(0 / 4) \\
-50 \% \text { peripheral } \\
(2 / 4)\end{array}$ & $\begin{array}{c}80 \%(4 / 5): \\
-0 \% \text { homogeneous } \\
(0 / 4) \\
-25 \% \text { peripheral }(1 / 4)\end{array}$ & $\begin{array}{c}71 \%(5 / 7): \\
-80 \% \text { homogeneous } \\
(4 / 5) \\
-0 \% \text { peripheral }(0 / 5)\end{array}$ & $\begin{array}{c}100 \%(1 / 1): \\
-100 \% \\
\text { heterogeneous }(1 / 1) \\
-100 \% \text { peripheral } \\
(1 / 1)\end{array}$ & $\begin{array}{c}100 \%(4 / 4): \\
-50 \% \text { heterogeneous } \\
(2 / 4) \\
-0 \% \text { peripheral }(0 / 4)\end{array}$ & $\begin{array}{c}100 \%(2 / 2): \\
-100 \% \\
\text { heterogeneous }(2 / 2) \\
-50 \% \text { peripheral } \\
(1 / 2)\end{array}$ & $\begin{array}{c}100 \%(1 / 1): \\
-100 \% \text { homogeneous } \\
(1 / 1) \\
-0 \% \text { peripheral }(0 / 1)\end{array}$ \\
\hline $\begin{array}{c}\text { Oedema surrounding } \\
\text { the lesion }\end{array}$ & $0 \%(0 / 4)$ & $0 \%(0 / 5)$ & $0 \%(0 / 7)$ & $100 \%(1 / 1)$ & $100 \%(4 / 4)$ & $100 \%(2 / 2)$ & $0 \%(0 / 1)$ \\
\hline $\begin{array}{l}\text { Dissection of nerve } \\
\text { fascicles }\end{array}$ & $0 \%(0 / 4)$ & $0 \%(0 / 5)$ & $0 \%(0 / 7)$ & $0 \%(0 / 1)$ & $100 \%(4 / 4)$ & $100 \%(2 / 2)$ & $0 \%(0 / 1)$ \\
\hline
\end{tabular}

NM: not measured (because shape not allowed reliable measurement)

NF1: neurofibromatosis type I

MPNST: malignant peripheral nerve sheath tumours 
[11]. In nerve sheath tumours, every category of lesion can be without the target sign. These latter sign is not strongly suggestive of malignant tumours, as described by some authors [12]. On the contrary, the target sign was present in any of the malignant lesions. Its presence is highly correlated with benignity; this agrees with the published sensitivity of $100 \%$ [13].

In our study, all the histological types had increased uptake after injection. This criterion cannot be used to distinguish between benign and malignant tumours: $82 \%$ of benign lesions and $100 \%$ of malignant lesions had increased uptake. Heterogeneous increased uptake is also not a differentiating criterion: none of the schwannomas or isolated neurofibromas had homogeneous uptake versus $50 \%$ of the lymphomas. The idea of using peripheral increased uptake as evidence of central necrosis has been described by some authors as a meaningful criterion for malignancy [14]. This was not true in our study population, as this sign was present in three of the benign tumours.

Invasion of soft tissues around the tumour is suggestive of a malignant lesion: in our study, none of benign lesions were accompanied by oedema of the soft tissues, while all the malignant lesions had this sign. This is widely accepted by the scientific community $[11,15]$.

Nerve fascicle dissection by tumour process has never been described and is suggestive of a malignant lesion of non-neurogenic origin: the MPNST in our study did not have this sign, in contrast to four cases of lymphoma and two cases of metastasis which did.

In our study, there were 4 schwannomas for 10 neurofibromas. Published studies indicate that each corresponds to $5 \%$ of benign tumours of peripheral nerve sheaths [15]. Neurofibromas more frequently affect the sciatic nerve than do schwannomas, which affect this nerve in only $1 \%$ of cases [14]. Schwannomas are more frequent in women $[16,17]$. This was consistent with our study $(3 / 4)$. The average age at the time of discovery was higher ( 50 years); the deep location of the sciatic nerve can lead to delayed diagnosis. As described by Kransdorf et al. [18], they present as fusiform tumours with regular borders, since $70 \%$ of them are encapsulated. Large tumours have heterogeneous gadolinium uptake, likewise in our study where the average size was $5 \mathrm{~cm}$. Schwannomas have been described as being offcentre to the carrier nerve [19]. This suggestive but not specific feature was found in $75 \%$ of our cases. The target sign was found in only one patient, versus $50 \%$ in published studies. One of the reasons may be its deep location: schwannomas of the sciatic nerve are discovered when they are larger, hence the signal is more heterogeneous.

Our study had more plexiform neurofibromas than isolated neurofibromas. This is probably due to our facility being a referral centre. The average size $(10.7 \mathrm{~cm})$ in our study is larger than published values [20]. However, it is similar to neurofibromas $(10.8 \mathrm{~cm})$ studied by Ogose et al., which were mainly located in the legs [15]. The target sign was present in $80 \%$ of isolated neurofibromas and $86 \%$ of plexiform neurofibromas in our study. This is consistent with published studies showing that this sign is more often associated with neurofibromas [21]. Hence, the target sign is not pathognomonic, as initially thought but highly specific (95\%) for benign lesions [22]. Our study found increased gadolinium uptake of neurofibromas in $66 \%$ of cases $(9 / 12)$, as in published results [22]. The centred nature of the tumour relative to the affected nerve is not pathognomonic of neurofibromas: one of the isolated neurofibromas in our study appeared centred. However, the location relative to the central axis of the nerve is suggestive of schwannomas when off-centred and suggestive of isolated neurofibroma when centred. The clinical context is crucial for isolated tumours.
While isolated neurofibromas are associated with NF1 in $10 \%$ of cases [18], a single benign nerve lesion in a patient affected by NF1 is a strong predictor: two patients in our study with a localised neurofibroma had NF1 versus zero for schwannomas.

Our study showed that benign tumours of the peripheral nerve sheaths such as schwannomas and neurofibromas have a fusiform shape that is in continuity with the nerve, with well-defined margins and no oedema surrounding the lesion. A positive target sign and the location relative to the nerve axis helps to orient the diagnosis but is not specific.

Our study shows that both MPNST and benign lesions have a fusiform shape and the nerve enters or exits the tumour. They can be differentiated based on the presence of ill-defined margins and the absence of the target sign. According to Van Herendael et al., ill-defined margins are suggestive of a malignant tumour that is not neurogenic, with MPNSTs having well-defined margins [12]. Heterogeneity with necrotic and haemorrhagic remodelling, while sometimes visible in large schwannomas, is suggestive of malignancy for neurogenic tumours according to some authors [2]. In our study, most schwannomas (3/4) and all the neurofibromas (5/5) were heterogeneous, given that they were discovered at a later stage. Peripheral uptake of contrast product was observed in our single case of MPNST, as described in other studies [14]; however, it was also observed in benign lesions.

Our study shows that the presence of ill-defined margins and invasion of soft tissue in the case of a neurogenic tumour are suggestive of MPNST. Conversely, our study shows that the size, signal and type of uptake are not indicative of malignant degeneration.

We studied three cases of lymph node involvement. This secondary involvement due to proximity occurs in $90 \%$ of cases [23]. Primary involvement is very rare $(0.2 \%)$ and only a few cases have been described. We found none in our study. These lesions present as irregular tumours in the soft tissues with greatly increased uptake associated with nerve thickening and fascicle dissection.

Metastases affecting peripheral nerves are rare and, in most cases, due to direct extension. Remote metastasis is very rare. Only four cases of metastatic lesions affecting the sciatic nerve have ever been described [24]. Our first case of endoneural metastasis occurred in a 59-year-old male with history of pleural mesothelioma. When the patient noticed a lump of his thigh, MRI found a lesion with heterogeneous gadolinium uptake that dissected the fascicles of the sciatic nerve and invaded the soft tissues. A biopsy confirmed the malignant tumour with fusiform cells; the immunohistochemical profile was suggestive of metastasis of mesothelioma. Our second case is identical to this described by Nagao et al. [25]: a metastatic lesion in the gluteal muscles was discovered with increased uptake of the entire sciatic nerve and adjacent soft tissues, secondary to bladder rupture during a transurethral resection of an invasive urothelial carcinoma. This suggests that the lumbar plexus was invaded by tumour cells after the bladder rupture. Both cases of metastasis had sciatic nerve fascicle dissection: these two lesions, which did not develop from axons or Schwann cells, extended into the supporting connective tissue found between the fascicles.

Intraneural perineurioma is a benign tumour caused by proliferation of perineural cells leading to localised nerve hypertrophy. This lesion was first described as its own tumour type in 1978 by Lazarus et al. [26]. It affects young adults and is typically located in the sciatic nerve. On MRI, it appears as segmental widening of the nerve trunk on T1 sequences, with T2 hyperintensity and increased gadolinium uptake. All these features were present in the female 
patient with this type of tumour. The lesion was $7 \mathrm{~cm}$ in height at the proximal portion of the sciatic nerve and was off-centred.

Our small number of patients, despite a 15 -year collection period at a referral centre, is evidence that tumours of the sciatic nerve are rare. Our study did not include signs that could be found on diffusionweighted and perfusion-weighted imaging; these sequences have only become routine in the past few years, not all patients were imaged with these methods over the study period.

\section{Conclusion}

Sciatic nerve tumours are deep lesions that most often require histological confirmation through a biopsy. Only plexiform neurofibromas have disease-specific signs on MRI. The clinical context will point to the aetiology: a benign neurogenic tumour in the context of NF1 is highly suggestive of neurofibroma. The presence of soft tissue oedema surrounding the lesion is suggestive of malignancy. Nerve fascicle dissection is not present in MPNSTs and is suggestive of non-neurogenic malignant origin such as lymph node infiltration or metastasis. The size, shape, signal and gadolinium uptake of sciatic nerve tumours cannot be used as diagnostic criteria.

\section{Acknowledgement}

The authors wish to thank Joanne Archambault, $\mathrm{PhD}$ for the editorial assistance provided during the preparation of this manuscript.

\section{Declaration of interest}

None

\section{References}

1. Kleihues P, Louis DN, Scheithauer BW, Rorke LB, Reifenberger G, et al. (2002) The WHO classification of tumors of the nervous system. J Neuropathol Exp Neurol 61: 215-225. [Crossref]

2. Murphey MD, Smith WS, Smith SE, Kransdorf MJ, Temple HT (1999) From the archives of the AFIP. Imaging of musculoskeletal neurogenic tumors: radiologicpathologic correlation. Radiographics 19: 1253-80. [Crossref]

3. Bhargava R, Parham DM, Lasater OE, Chari RS, Chen G, et al. (1997) MR imaging differentiation of benign and malignant peripheral nerve sheath tumors: use of the target sign. Pediatr Radiol 27: 124-129. [Crossref]

4. Lin J, Martel W (2001) Cross-sectional imaging of peripheral nerve sheath tumors: characteristic signs on CT, MR imaging, and sonography. AJR Am J Roentgenol 176: 75-82. [Crossref]

5. Amrami KK, Felmlee JP, Spinner RJ (2008) MRI of peripheral nerves. Neurosurg Clin NAm 19: 559-572, vi. [Crossref]

6. Furniss D, Swan MC, Morritt DG, Lim J, Khanna T, et al. (2008) A 10-year review of benign and malignant peripheral nerve sheath tumors in a single center: clinical and radiographic features can help to differentiate benign from malignant lesions. Plast Reconstr Surg 121: 529-33. [Crossref]

7. Landis JR, Koch GG (1977) The measurement of observer agreement for categorical data. Biometrics 33: 159-174. [Crossref]
8. Mautner VF, Friedrich RE, von Deimling A, Hagel C, Korf B, et al. (2003) Malignant peripheral nerve sheath tumours in neurofibromatosis type 1: MRI supports the diagnosis of malignant plexiform neurofibroma. Neuroradiology 45: 618-25. [Crossref]

9. Ogose A, Hotta T, Morita T, Yamamura S, Hosaka N, et al. (1999) Tumors of peripheral nerves: correlation of symptoms, clinical signs, imaging features, and histologic diagnosis. Skeletal Radiol 28: 183-8. [Crossref]

10. Wasa J, Nishida Y, Tsukushi S, Shido Y, Sugiura H, et al. (2010) MRI features in the differentiation of malignant peripheral nerve sheath tumors and neurofibromas. AJR Am J Roentgenol 194: 1568-74. [Crossref]

11. Pilavaki M, Chourmouzi D, Kiziridou A, Skordalaki A, Zarampoukas T, et al. (2004) Imaging of peripheral nerve sheath tumors with pathologic correlation: pictorial review. Eur J Radiol 52: 229-39. [Crossref]

12. Van Herendael BH, Heyman SRG, Vanhoenacker FM, De Temmerman G, Bloem JL, et al. (2006) The value of magnetic resonance imaging in the differentiation between malignant peripheral nerve-sheath tumors and non-neurogenic malignant soft-tissue tumors. Skeletal Radiol 35: 745-53. [Crossref]

13. Li CS, Huang GS, Wu HD, Chen WT, Shih LS, et al. (2008) Differentiation of soft tissue benign and malignant peripheral nerve sheath tumors with magnetic resonance imaging. Clin Imaging 32: 121-7. [Crossref]

14. Wasa J, Nishida Y, Tsukushi S, Shido Y, Sugiura H, et al. (2010) MRI features in the differentiation of malignant peripheral nerve sheath tumors and neurofibromas. AJR Am J Roentgenol 194: 1568-1574. [Crossref]

15. Ogose A, Hotta T, Morita T, Yamamura S, Hosaka N, et al. (1999) Tumors of peripheral nerves: correlation of symptoms, clinical signs, imaging features, and histologic diagnosis. Skeletal Radiol 28: 183-8. [Crossref]

16. Bianchi S (2008) Ultrasound of the peripheral nerves. Joint Bone Spine 75: 643-649. [Crossref]

17. Woodruff JM (1993) The pathology and treatment of peripheral nerve tumors and tumor-like conditions. CA Cancer J Clin 43: 290-308. [Crossref]

18. Kransdorf MJ (1995) Benign soft-tissue tumors in a large referral population: distribution of specific diagnoses by age, sex, and location. AJR Am J Roentgenol 164 395-402. [Crossref]

19. Gominak SC, Ochoa JL (1998) Sciatic schwannoma of the thigh causing foot pain mimicking plantar neuropathy. Muscle Nerve 21: 528-30. [Crossref]

20. Furniss D, Swan MC, Morritt DG, Lim J, Khanna T, et al. (2008) A 10-year review of benign and malignant peripheral nerve sheath tumors in a single center: clinical and radiographic features can help to differentiate benign from malignant lesions. Plast Reconstr Surg 121: 529-33. [Crossref]

21. Kransdorf MJ, Murphey MD (2006) Imaging of Soft Tissue Tumors. Lippincott Williams \& Wilkins; 607.

22. Jee WH, Oh SN, McCauley T, Ryu KN, Suh JS, et al. (2004) Extraaxial neurofibromas versus neurilemmomas: discrimination with MRI. AJR Am J Roentgenol 183: 629-33. [Crossref]

23. Kanamori M, Matsui H, Yudoh K (1995) Solitary T-cell lymphoma of the sciatic nerve: case report. Neurosurgery 36: 1203-1205. [Crossref]

24. Varin S, Faure A, Bouc P, Maugars Y, Berthelot JM (2006) Endoneural metastasis of the sciatic nerve disclosing the relapse of a renal carcinoma, four years after its surgical treatment. Joint Bone Spine 73: 760-2. [Crossref]

25. Nagao E, Nishie A, Yoshimitsu K, Irie H, Shioyama Y, et al. (2004) Gluteal muscular and sciatic nerve metastases in advanced urinary bladder carcinoma: case report. Abdom Imaging 29: 619-22. [Crossref]

26. Lazarus SS, Trombetta LD (1978) Ultrastructural identification of a benign perineurial cell tumor. Cancer 41: 1823-9. [Crossref]

Copyright: (C2019 Molinier B. This is an open-access article distributed under the terms of the Creative Commons Attribution License, which permits unrestricted use, distribution, and reproduction in any medium, provided the original author and source are credited. 